\title{
A NOTE ON SUMS OF A CLASS OF SERIES
}

\author{
SUNGTAE JUN, GRADIMIR V. MILOVANOVIĆ, INSUK KIM, \\ AND ARJUN K. RATHIE
}

Received 03 November, 2018

Abstract. The aim of this note is to provide sums of a unified class of series of the form

$$
S_{i}(a)=\sum_{k=0}^{\infty}(-1)^{k}\left(\begin{array}{c}
a-i \\
k
\end{array}\right) \frac{1}{2^{k}(a+k+1)}
$$

in the most general form for any $i \in \mathbb{Z}$. For each $v \in \mathbb{N}$, in four cases when $i= \pm 2 v$ and $i= \pm(2 v-1)$, simple explicit expressions for $S_{i}(a)$ are obtained, e.g.

$$
S_{2 v}(a)=\frac{2^{2 v-1-a}}{(a-2 v+1)_{v}}\left[\frac{\sqrt{\pi} \Gamma(a+1)}{\Gamma\left(a+\frac{3}{2}-v\right)}-P_{v-1}(a)\right],
$$

where $P_{\nu}(a)$ is an algebraic polynomial in $a$ of degree $v$.

For $i=1$ and $a=n(\in \mathbb{N})$, we recover the well known sum of the series due to Vowe and Seiffert. Several other known results due to Srivastava and Kim et al. can be considered as special cases of our result.

2010 Mathematics Subject Classification: 33C15; 33C90; 05A19; 33B15; 33C05; 39A10

Keywords: hypergeometric series, Bailey summation theorem, generalization of Bailey summation theorem

\section{INTRODUCTION AND PRELIMINARIES}

In 1812, Gauss [6] defined his famous infinite series as follows:

$$
1+\frac{a \cdot b}{c} \frac{z}{1 !}+\frac{a(a+1) b(b+1)}{c(c+1)} \frac{z^{2}}{2 !}+\cdots .
$$

The series (1.1) is denoted by the notation

$$
{ }_{2} F_{1}\left[\begin{array}{c}
a, b \\
c
\end{array} ;\right], \text { or } \quad{ }_{2} F_{1}\left[\begin{array}{c}
a, b ; z \\
c ;
\end{array}\right] \text {, or } \quad{ }_{2} F_{1}[a, b ; c ; z],
$$

or simply $F$ and is popularly known as the Gauss's function or hypergeometric function.

The quantities $a, b$ and $c$ are known as the parameters (real or complex) of the series with $c \neq 0,-1,-2, \ldots$ and $z$ is termed as the variable of the series. This is 
called the 'Hypergeometric series' because either $a=1$ and $b=c$ or $b=1$ and $a=c$, it reduces to the well known 'Geometric series'.

In terms of Pochhammer's symbol $(a)_{n}$, defined by

$$
(a)_{n}= \begin{cases}a(a+1) \cdots(a+n-1), & n \in \mathbb{N}, \\ 1, & n=0,\end{cases}
$$

the series (1.1) is represented as

$$
\sum_{n=0}^{\infty} \frac{(a)_{n}(b)_{n}}{(c)_{n}} \cdot \frac{z^{n}}{n !}
$$

Also, in term of Gamma function, $(a)_{n}$ can be represented as

$$
(a)_{n}=\frac{\Gamma(a+n)}{\Gamma(a)} \text {. }
$$

Thus, from (1.1), we have

$$
{ }_{2} F_{1}\left[\begin{array}{c}
a, b \\
c
\end{array} ; z\right]=\sum_{n=0}^{\infty} \frac{(a)_{n}(b)_{n}}{(c)_{n}} \cdot \frac{z^{n}}{n !} .
$$

Here, we verify that the series (1.1)

(i) is convergent for all values of $z$ provided $|z|<1$ and divergent when $|z|>1$.

(ii) is convergent for $z=1$ provided $\operatorname{Re}(c-a-b)>0$ and divergent for $\operatorname{Re}(c-$ $a-b) \leq 0$.

(iii) is absolutely convergent for $z=-1$ provided $\operatorname{Re}(c-a-b)>0$ and convergent but not absolutely for $-1<\operatorname{Re}(c-a-b) \leq 0$ and divergent for $\operatorname{Re}(c-a-b)<-1$.

The limiting case of (1.1) is worth mentioning here. For this, if we replace $z$ by $z / b$ in (1.3) and take the limit as $b \rightarrow \infty$, then since

$$
\frac{(b)_{n}}{b^{n}} z^{n} \rightarrow z^{n},
$$

we arrive at the following series which is in the literature known as the Kummer's series or the confluent hypergeometric series [9]

$$
{ }_{1} F_{1}\left[\begin{array}{l}
a \\
c
\end{array} ; z\right]=\sum_{n=0}^{\infty} \frac{(a)_{n}}{(c)_{n}} \frac{z^{n}}{n !} .
$$

Further, it is interesting to mention here that almost all elementary functions of mathematics and mathematical physics are special cases or limiting cases of Gauss's hypergeometric function or the confluent hypergeometric function, see [3, 15].

It is well known that whenever a ${ }_{2} F_{1}$ hypergeometric function reduces to a gamma function, then the obtained result is very useful for application. Thus, for example, 
Gauss summation theorem, Gauss second, Kummer, Vandermonde and Bailey's theorems for the series ${ }_{2} F_{1}$ play a vital role in the theory of hypergeometric function, especially in applied mathematics, mathematical physics and engineering.

However, in this note, we are interested in the following Bailey's summation theorem [1,2] viz.

$$
{ }_{2} F_{1}\left[\begin{array}{c}
a, 1-a \\
b
\end{array} ; \frac{1}{2}\right]=\frac{\Gamma\left(\frac{1}{2} b\right) \Gamma\left(\frac{1}{2} b+\frac{1}{2}\right)}{\Gamma\left(\frac{1}{2} b+\frac{1}{2} a\right) \Gamma\left(\frac{1}{2} b-\frac{1}{2} a+\frac{1}{2}\right)} .
$$

During 1992-96, Lavoie et al. [10-12] have successfully investigated the generalizations of the above mentioned classical summation theorems and also obtained the generalization of the classical summation theorems for the series ${ }_{3} F_{2}$ with unit argument such as those of Watson, Dixon and Whipple.

In particular, they have generalized Bailey's summation theorem (1.5) by obtaining a single formula containing eleven results in the form

$$
{ }_{2} F_{1}\left[\begin{array}{cc}
a, 1-a+i & ; \frac{1}{2}
\end{array}\right]
$$

for $i=0, \pm 1, \pm 2, \pm 3, \pm 4, \pm 5$. Later, Rakha and Rathie [17] and Kim et al. [8] have further generalized and extended respectively, the above mentioned classical summation theorems in the most general form.

One of such kind of summation theorems will be used in this note in order to provide sums of a unified class of the series of the form

$$
\sum_{k=0}^{\infty}(-1)^{k}\left(\begin{array}{c}
a-i \\
k
\end{array}\right) \frac{1}{2^{k}(a+k+1)}
$$

in the most general form for any $i \in \mathbb{Z}$. Otherwise, the first result of this type was appeared in 1987, when Vowe and Seiffert [20] evaluated the following very interesting and useful sum, viz.

$$
\sum_{k=0}^{n-1}(-1)^{k}\left(\begin{array}{c}
n-1 \\
k
\end{array}\right) \frac{1}{2^{k}(n+k+1)}=\frac{2^{n}(n-1) ! n !}{(2 n) !}-\frac{1}{2^{n} n} \quad(n \in \mathbb{N})
$$

by identifying it with an Eulerian integral of the type

$$
\int_{0}^{1}\left(1-\frac{t}{2}\right)^{n-1} t^{n} \mathrm{~d} t
$$

In recent years, the sum (1.8) has gained a fair amount of attention. In 1988, Srivastava [18] established the generalization of (1.8) in the form

$$
\sum_{k=0}^{\infty}(-1)^{k}\left(\begin{array}{c}
a-1 \\
k
\end{array}\right) \frac{1}{2^{k}(a+k+1)}=\frac{2^{a} \Gamma(a) \Gamma(a+1)}{\Gamma(2 a+1)}-\frac{1}{2^{a} a}
$$


by employing the classical Bailey's summation theorem (1.5) and also discussed a few more series of this type.

In 1989, Srivastava [19] gave a basic (or $q-$ ) extension of (1.10).

In 1999, Choi et al. [4] have re-derived the result (1.10) and obtained the following two results closely related to (1.10) by utilizing certain contiguous function relations for ${ }_{2} F_{1}$ due to Gauss $[6,16]$,

$$
\sum_{k=0}^{\infty}(-1)^{k}\left(\begin{array}{l}
a \\
k
\end{array}\right) \frac{k}{2^{k}(a+k)(a+k+1)}=\frac{2^{a-1}\{\Gamma(a+1)\}^{2}}{\Gamma(2 a+2)}-\frac{1}{2^{a+1}}
$$

and

$$
\sum_{k=0}^{\infty}(-1)^{k}\left(\begin{array}{c}
a-2 \\
k
\end{array}\right) \frac{k}{2^{k}(a+k)(a+k+1)}=\frac{3 \cdot 2^{a}\{\Gamma(a+1)\}^{2}}{(a-1) \Gamma(2 a+1)}-\frac{a+2}{(a-1)^{2 a-1}} .
$$

In 2009, Prodinger [14] established a more general sum of the form

$$
\sum_{k=0}^{n}(-1)^{k}\left(\begin{array}{l}
n \\
k
\end{array}\right) \frac{1}{2^{k}(m+k)} \quad(m, k \in \mathbb{N})
$$

and obtained as special cases, the results (1.11) and (1.12) for $a=n$ by completely elementary tools.

In 2010, Dahlberg et al. [5] have presented an elegant and elementary proof of the identites (1.11) and (1.12) for $a=n$ using Zeilberger algorithm and the WilfZeilberger proof style.

In 2012, Kim et al. [7] generalized the sum (1.10) (and of course, Vowe and Seiffert's sum (1.8)) and obtained the explicit expressions of (1.7) for $i=0, \pm 1, \pm 2$, $\pm 3, \pm 4, \pm 5$ by utilizing the generalizations of the Bailey's summation formulas (1.6) obtained earlier by Lavoie et al. [11] and deduced a large number of very interesting results including (1.8) and (1.10).

In our present investigation, we will use the generalization of the Bailey's summation theorem (1.5) in the following two forms [17]:

$$
\begin{aligned}
{ }_{2} F_{1}\left[\begin{array}{c}
a, 1-a+i \\
b
\end{array} ; \frac{1}{2}\right]= & \frac{2^{1+i-b} \Gamma\left(\frac{1}{2}\right) \Gamma(b) \Gamma(a-i)}{\Gamma(a) \Gamma\left(\frac{1}{2} b-\frac{1}{2} a\right) \Gamma\left(\frac{1}{2} b-\frac{1}{2} a+\frac{1}{2}\right)} \\
& \times \sum_{r=0}^{i}\left(\begin{array}{l}
i \\
r
\end{array}\right) \frac{(-1)^{r} \Gamma\left(\frac{1}{2} b-\frac{1}{2} a+\frac{1}{2} r\right)}{\Gamma\left(\frac{1}{2} b+\frac{1}{2} a+\frac{1}{2} r-i\right)}
\end{aligned}
$$

for $i=0,1,2, \ldots$ and

$$
{ }_{2} F_{1}\left[\begin{array}{cc}
a, 1-a-i & ; \frac{1}{2}
\end{array}\right]=\frac{2^{1-i-b} \Gamma\left(\frac{1}{2}\right) \Gamma(b)}{\Gamma\left(\frac{1}{2} b-\frac{1}{2} a\right) \Gamma\left(\frac{1}{2} b-\frac{1}{2} a+\frac{1}{2}\right)}
$$




$$
\times \sum_{r=0}^{i}\left(\begin{array}{l}
i \\
r
\end{array}\right) \frac{\Gamma\left(\frac{1}{2} b-\frac{1}{2} a+\frac{1}{2} r\right)}{\Gamma\left(\frac{1}{2} b+\frac{1}{2} a+\frac{1}{2} r\right)}
$$

for $i=0,1,2, \ldots$, as well as a general formula (containing (1.13) and (1.14)), obtained recently by Milovanović et al. [13],

$$
\begin{aligned}
& { }_{2} F_{1}\left[\begin{array}{cc}
a, 1-a+i & ; \frac{1}{2}
\end{array}\right] \\
& =\frac{\Gamma\left(\frac{1}{2}\right) \Gamma(b) \Gamma(1-a)}{2^{b-i-1} \Gamma\left(1-a+\frac{1}{2}(i+|i|)\right)}\left\{\frac{C_{i}(a, b)}{\Gamma\left(\frac{1}{2} b-\frac{1}{2} a+\frac{1}{2}\right) \Gamma\left(\frac{1}{2} b+\frac{1}{2} a-\left\lfloor\frac{1+i}{2}\right\rfloor\right)}\right. \\
& \left.+\frac{D_{i}(a, b)}{\Gamma\left(\frac{1}{2} b-\frac{1}{2} a\right) \Gamma\left(\frac{1}{2} b+\frac{1}{2} a-\frac{1}{2}-\left\lfloor\frac{i}{2}\right\rfloor\right)}\right\},
\end{aligned}
$$

with coefficients $C_{i}(a, b)$ and $D_{i}(a, b)$ given by

$$
\begin{aligned}
C_{2 v}(a, b) & =\sum_{j=0}^{v}\left(\begin{array}{c}
2 v \\
2 j
\end{array}\right)\left(\frac{b-a}{2}\right)_{j}\left(\frac{b+a}{2}-(2 v-j)\right)_{v-j}, \\
D_{2 v}(a, b) & =-\sum_{j=0}^{v-1}\left(\begin{array}{c}
2 v \\
2 j+1
\end{array}\right)\left(\frac{b-a+1}{2}\right)_{j}\left(\frac{b+a+1}{2}-(2 v-j)\right)_{v-j-1}, \\
C_{2 v+1}(a, b) & =-\sum_{j=0}^{v}\left(\begin{array}{c}
2 v+1 \\
2 j
\end{array}\right)\left(\frac{b-a}{2}\right)_{j}\left(\frac{b+a}{2}-(2 v-j+1)\right)_{v-j}, \\
D_{2 v+1}(a, b) & =\sum_{j=0}^{v}\left(\begin{array}{c}
2 v+1 \\
2 j+1
\end{array}\right)\left(\frac{b-a+1}{2}\right)_{j}\left(\frac{b+a+1}{2}-(2 v-j+1)\right)_{v-j} \\
C_{-2 v}(a, b) & =\sum_{j=0}^{v}\left(\begin{array}{c}
2 v \\
2 j
\end{array}\right)\left(\frac{b-a}{2}\right)_{j}\left(\frac{b+a}{2}+j\right)_{v-j}, \\
D_{-2 v}(a, b) & =\sum_{j=0}^{v-1}\left(\begin{array}{c}
2 v \\
2 j+1
\end{array}\right)\left(\frac{b-a+1}{2}\right)_{j}\left(\frac{b+a+1}{2}+j\right)_{v-j-1} \\
C_{-(2 v+1)}(a, b) & =\sum_{j=0}^{v}\left(\begin{array}{c}
2 v+1 \\
2 j
\end{array}\right)\left(\frac{b-a}{2}\right)_{j}\left(\frac{b+a}{2}+j\right)_{v-j}
\end{aligned}
$$




$$
D_{-(2 v+1)}(a, b)=\sum_{j=0}^{v}\left(\begin{array}{l}
2 v+1 \\
2 j+1
\end{array}\right)\left(\frac{b-a+1}{2}\right)_{j}\left(\frac{b+a+1}{2}+j\right)_{v-j} .
$$

Clearly, for $i=0$, the results (1.13), (1.14) and (1.15) reduce to the Bailey's summation theorem (1.5).

The main objective of this note is to provide sums of a unified class of the series of the form

$$
S_{i}(a)=\sum_{k=0}^{\infty}(-1)^{k}\left(\begin{array}{c}
a-i \\
k
\end{array}\right) \frac{1}{2^{k}(a+k+1)}
$$

in the most general form for any $i \in \mathbb{Z}$. The results obtained earlier by Vowe and Seiffert [20] and Srivastava [18] follows as special cases of our main findings.

\section{MAIN RESULTS}

The following theorem gives a result on sums of a unified class of series.

Theorem 1. For $i=0,1,2, \cdots$, the following results hold true.

$$
\begin{aligned}
\sum_{k=0}^{\infty}(-1)^{k}\left(\begin{array}{c}
a-i \\
k
\end{array}\right) & \frac{1}{2^{k}(a+k+1)} \\
& =\frac{\Gamma(a+1-i)}{2^{a-i+1}} \sum_{r=0}^{i}(-1)^{r}\left(\begin{array}{l}
i \\
r
\end{array}\right) \frac{\Gamma\left(\frac{1}{2} r+\frac{1}{2}\right)}{\Gamma\left(a+\frac{3}{2}+\frac{1}{2} r-i\right)}
\end{aligned}
$$

and

$$
\sum_{k=0}^{\infty}(-1)^{k}\left(\begin{array}{c}
a+i \\
k
\end{array}\right) \frac{1}{2^{k}(a+k+1)}=\frac{\Gamma(a+1)}{2^{a+i+1}} \sum_{r=0}^{i}\left(\begin{array}{l}
i \\
r
\end{array}\right) \frac{\Gamma\left(\frac{1}{2} r+\frac{1}{2}\right)}{\Gamma\left(a+\frac{3}{2}+\frac{1}{2} r\right)} .
$$

Proof. The proofs of our results are quite straight forward. For this, in order to establish the result (2.1), we consider (1.16) for $i \in \mathbb{N}_{0}$.

If we use the elementary identities

$$
\left(\begin{array}{l}
n \\
r
\end{array}\right)=\frac{n !}{(n-r) ! r !}, \quad(a)_{n}=\frac{\Gamma(a+n)}{\Gamma(a)}, \quad \Gamma(\alpha-n)=\frac{(-1)^{n} \Gamma(\alpha)}{(1-\alpha)_{n}},
$$

then, after some simplification, the left-hand side in (2.1) becomes

$$
S_{i}(a)=\frac{1}{a+1} \sum_{k=0}^{\infty} \frac{(-a+i)_{k}(a+1)_{k}}{(a+2)_{k}} \cdot \frac{1}{k ! 2^{k}}
$$

Summing up the series with the help of (1.3), we get

$$
S_{i}(a)=\frac{1}{a+1}{ }_{2} F_{1}\left[\begin{array}{c}
-a+i, a+1 \\
a+2
\end{array} ; \frac{1}{2}\right] .
$$


We now observe here that the right-hand side of (2.1) can be verified with the result (1.13) to ${ }_{2} F_{1}$ above and some calculation.

In exactly the same manner, the second result (2.2) for $S_{-i}(a), i \in \mathbb{N}$, can be proven with the help of the known result (1.14).

Alternatively, the results given in previous theorem can be expressed in the following explicit form:

Theorem 2. For each $v \in \mathbb{N}$ the sum (1.16) can be expressed as

$$
S_{2 v}(a)=\frac{2^{2 v-1-a}}{(a-2 v+1)_{v}}\left[\frac{\sqrt{\pi} \Gamma(a+1)}{\Gamma\left(a+\frac{3}{2}-v\right)}-P_{v-1}(a)\right]
$$

and

$$
S_{2 v-1}(a)=\frac{2^{2 v-2-a}}{(a-2 v+2)_{v}}\left[\frac{\sqrt{\pi} \Gamma(a+1)}{\Gamma\left(a+\frac{3}{2}-v\right)}-Q_{v-1}(a)\right],
$$

where $P_{v}$ and $Q_{v}$ are polynomials of degree $v$, defined by the recurrence relations

$$
\left.\begin{array}{l}
P_{v}(a)=2 Q_{v}(a-1)-(a-2 v-1) P_{\nu-1}(a-1), \\
Q_{\nu}(a)=2(a-v) P_{\nu-1}(a-1)-(a-2 v) Q_{v-1}(a-1),
\end{array}\right\}
$$

with $P_{0}(a)=2$ and $Q_{0}(a)=1$.

For negative indices we have

$$
S_{-2 v}(a)=\frac{2^{-a-2 v-1}}{(a+1)_{v}}\left[\frac{\sqrt{\pi} \Gamma(a+2 v+1)}{\Gamma\left(a+\frac{3}{2}+v\right)}+R_{v-1}(a)\right]
$$

and

$$
S_{-(2 v-1)}(a)=\frac{2^{-a-2 v}}{(a+1)_{v}}\left[\frac{\sqrt{\pi} \Gamma(a+2 v)}{\Gamma\left(a+\frac{1}{2}+v\right)}+T_{\nu-1}(a)\right],
$$

where $R_{v}$ and $T_{v}$ are polynomials of degree $v$, defined by the recurrence relations

$$
\left.\begin{array}{l}
T_{\nu}(a)=2(a+v+1) R_{\nu-1}(a)-(a+1) T_{\nu-1}(a+1), \\
R_{\nu}(a)=2 T_{\nu}(a)-(a+1) R_{\nu-1}(a+1),
\end{array}\right\}
$$

with $R_{0}(a)=2$ and $T_{0}(a)=1$.

The expressions (2.6) and (2.9) hold also for $v=0$ if we take $P_{-1}(a)=R_{-1}(a)=$ 0 .

In proving Theorem 2.2 we need the following auxiliary result: 
Lemma 1. For each $v \in \mathbb{N}_{0}$ the sum (1.16) satisfies the following recurrence relations

$$
\left.\begin{array}{rl}
S_{2 v+1}(a) & =2\left[S_{2 v}(a-1)-S_{2 v-1}(a-1)\right], \\
S_{2 v+2}(a) & =2\left[S_{2 v+1}(a-1)-S_{2 v}(a-1)\right], \\
S_{-(2 v+1)}(a) & =S_{-2 v}(a)-\frac{1}{2} S_{-(2 v-1)}(a+1), \\
S_{-(2 v+2)}(a) & =S_{-(2 v+1)}(a)-\frac{1}{2} S_{-2 v}(a+1) .
\end{array}\right\}
$$

Proof. Starting from (1.16) for $i=2 v+1$ and using the identity

$$
\left(\begin{array}{c}
a-2 v-1 \\
k
\end{array}\right)=\left(\begin{array}{c}
a-2 v \\
k+1
\end{array}\right)-\left(\begin{array}{c}
a-2 v-1 \\
k+1
\end{array}\right),
$$

we can easily prove the first recurrence relation in (2.10). In a similar way we get the other ones. We omit the details.

Proof of Theorem 2. In order to prove explicit expressions (2.4), (2.5), (2.7), and (2.8), we apply (1.15) to (2.3) for $i=2 v, 2 v-1,-2 v$, and $-(2 v-1)$, respectively, and use the corresponding coefficients $C_{i}(a, b)$ and $D_{i}(a, b)$.

Finally, using Lemma 1 we obtain the recurrence relations (2.6) and (2.9) for the polynomials $P_{v}$ and $Q_{v}$ and $R_{v}$ and $T_{v}$, respectively.

Remark 1. The polynomials $P_{v}$ and $Q_{v}$ which satisfy the recurrence relations (2.6) are given, for $v=0,1, \ldots, 9$, by

$$
\begin{aligned}
P_{0}(a)= & 2, \quad P_{1}(a)=4(a-1), \quad P_{2}(a)=2\left(3 a^{2}-11 a+12\right), \\
P_{3}(a)= & 8(a-3)\left(a^{2}-5 a+10\right), \\
P_{4}(a)= & 2\left(5 a^{4}-70 a^{3}+427 a^{2}-1322 a+1680\right), \\
P_{5}(a)= & 4(a-5)\left(3 a^{4}-50 a^{3}+401 a^{2}-1698 a+3024\right), \\
P_{6}(a)= & 2\left(7 a^{6}-217 a^{5}+3227 a^{4}-28967 a^{3}+159750 a^{2}\right. \\
& -496680 a+665280), \\
P_{7}(a)= & 16(a-7)\left(a^{6}-35 a^{5}+623 a^{4}-6889 a^{3}+47256 a^{2}\right. \\
& -183516 a+308880), \\
P_{8}(a)= & 6\left(3 a^{8}-164 a^{7}+4494 a^{6}-79280 a^{5}+953387 a^{4}-7765436 a^{3}\right. \\
& \left.+40967236 a^{2}-126332400 a+172972800\right), \\
P_{9}(a)= & 4(a-9)\left(5 a^{8}-300 a^{7}+9402 a^{6}-193944 a^{5}+2747829 a^{4}\right. \\
& \left.-26422764 a^{3}+164805772 a^{2}-602206800 a+980179200\right),
\end{aligned}
$$


and

$$
\begin{aligned}
Q_{0}(a)= & 1, Q_{1}(a)=3 a-2, \quad Q_{2}(a)=5 a^{2}-15 a+12, \\
Q_{3}(a)= & 7 a^{3}-49 a^{2}+126 a-120 \\
Q_{4}(a)= & 3\left(3 a^{4}-38 a^{3}+201 a^{2}-526 a+560\right) \\
Q_{5}(a)= & 11 a^{5}-220 a^{4}+1969 a^{3}-9812 a^{2}+26532 a-30240, \\
Q_{6}(a)= & 13 a^{6}-377 a^{5}+5109 a^{4}-41119 a^{3}+202046 a^{2} \\
& -558792 a+665280, \\
Q_{7}(a)= & 15 a^{7}-595 a^{6}+11361 a^{5}-134185 a^{4}+1032120 a^{3}-5031580 a^{2} \\
& +14102064 a-17297280, \\
Q_{8}(a)= & 17 a^{8}-884 a^{7}+22610 a^{6}-367880 a^{5}+4060433 a^{4}-30316916 a^{3} \\
& +146566860 a^{2}-414018000 a+518918400, \\
Q_{9}(a)= & 19 a^{9}-1254 a^{8}+41382 a^{7}-886692 a^{6}+13256091 a^{5} \\
& -139709166 a^{4}+1017764108 a^{3}-4878321288 a^{2} \\
& +13847306400 a-17643225600,
\end{aligned}
$$

respectively.

Remark 2. The polynomials $R_{v}$ and $T_{v}$ which satisfy the recurrence relations (2.9) are given, for $v=0,1, \ldots, 9$, by

$$
\begin{aligned}
R_{0}(a)= & 2, \quad R_{1}(a)=4(a+3), \quad R_{2}(a)=2\left(3 a^{2}+25 a+54\right), \\
R_{3}(a)= & 8(a+5)\left(a^{2}+11 a+34\right), \\
R_{4}(a)= & 2\left(5 a^{4}+130 a^{3}+1327 a^{2}+6218 a+11160\right), \\
R_{5}(a)= & 4(a+7)\left(3 a^{4}+94 a^{3}+1193 a^{2}+7062 a+16200\right), \\
R_{6}(a)= & 2\left(7 a^{6}+371 a^{5}+8617 a^{4}+110585 a^{3}+817288 a^{2}\right. \\
& +3270524 a+5504688), \\
R_{7}(a)= & 16(a+9)\left(a^{6}+61 a^{5}+1663 a^{4}+25303 a^{3}+222952 a^{2}\right. \\
& +1067812 a+2158800), \\
R_{8}(a)= & 6\left(3 a^{8}+268 a^{7}+11046 a^{6}+269992 a^{5}+4228307 a^{4}\right. \\
& \left.+43076572 a^{3}+277195204 a^{2}+1026169008 a+1668885120\right), \\
R_{9}(a)= & 4(a+11)\left(5 a^{8}+500 a^{7}+23402 a^{6}+654296 a^{5}+11765429 a^{4}\right.
\end{aligned}
$$




$$
\left.+137947556 a^{3}+1023509532 a^{2}+4376435280 a+8236080000\right),
$$

and

$$
\begin{aligned}
T_{0}(a)= & 1, \quad T_{1}(a)=3 a+7, \quad T_{2}(a)=5 a^{2}+35 a+62, \\
T_{3}(a)= & 7 a^{3}+98 a^{2}+469 a+762, \\
T_{4}(a)= & 3\left(3 a^{4}+70 a^{3}+633 a^{2}+2606 a+4088\right), \\
T_{5}(a)= & 11 a^{5}+385 a^{4}+5599 a^{3}+41855 a^{2}+159390 a+245640, \\
T_{6}(a)= & 13 a^{6}+637 a^{5}+13559 a^{4}+158639 a^{3}+1065636 a^{2} \\
& +3868332 a+5897520 \\
T_{7}(a)= & 15 a^{7}+980 a^{6}+28686 a^{5}+481640 a^{4}+4958895 a^{3} \\
& +31072820 a^{2}+109138164 a+165145680, \\
T_{8}(a)= & 17 a^{8}+1428 a^{7}+54978 a^{6}+1250520 a^{5}+18186753 a^{4} \\
& +171842052 a^{3}+1024655212 a^{2}+3512174160 a+5284782720, \\
T_{9}(a)= & 19 a^{9}+1995 a^{8}+97698 a^{7}+2888646 a^{6}+56212203 a^{5} \\
& +740755755 a^{4}+6574624112 a^{3}+37758440004 a^{2} \\
& +126997604208 a+190253266560,
\end{aligned}
$$

respectively.

\section{CONCLUDING REMARK}

In this note, an attempt has been made to provide a unified sum of the series of the form

$$
\sum_{k=0}^{\infty}(-1)^{k}\left(\begin{array}{c}
a-i \\
k
\end{array}\right) \frac{1}{2^{k}(a+k+1)}
$$

in the most general form for any $i=0, \pm 1, \pm 2, \ldots$.

We believe that the results obtained in this note may be potentially useful in combinatorics, applied mathematics, mathematical physics and engineering.

\section{REFERENCES}

[1] G. E. Andrews, R. Askey, and R. Roy, Special functions. Cambridge: Cambridge University Press, 1999, vol. 71.

[2] W. N. Bailey, "Generalized hypergeometric series." Cambridge Tracts in Mathematics and Mathematical Physics. 32. London: Cambridge University Press. vii, 108 p. (1935)., 1935.

[3] Y. A. Brychkov, Handbook of special functions. Derivatives, integrals, series and other formulas. Boca Raton, FL: Chapman \& Hall/CRC, 2008. 
[4] J. Choi, P. Zornig, and A. K. Rathie, "Sums of certain classes of series." Commun. Korean Math. Soc., vol. 14, no. 3, pp. 641-647, 1999.

[5] S. Dahlberg, T. Ferdinands, and A. Tefera, "A Wilf-Zeilberger approach to sums of Choi, Zorig and Rathie." Quaest. Math., vol. 33, no. 3, pp. 341-346, 2010, doi: 10.2989/16073606.2010.507325.

[6] C. F. Gauss, "Disquisitiones generales circa serium infinitum, Thesis, Gottingen," Ges. Werke Gottingen, Vol. II, 437-445; III 123-163, III 207-229, III 446-460, 1866.

[7] Y. S. Kim, M. P. Chaudhary, and A. K. Rathie, "On sums of certain classes of series." Commun. Korean Math. Soc., vol. 27, no. 4, pp. 745-751, 2012, doi: 10.4134/CKMS.2012.27.4.745.

[8] Y. S. Kim, M. A. Rakha, and A. K. Rathie, "Extensions of certain classical summation theorems for the series ${ }_{2} F_{1},{ }_{3} F_{2}$ and with applications in Ramanujan's summations." Int. J. Math. Math. Sci., vol. 2010, p. 26, 2010.

[9] E. E. Kummer, "Uber die hypergeometrische Reihe $1+\frac{\alpha \cdot \beta}{1 \cdot \gamma} x+\frac{\alpha(\alpha+1) \beta(\beta+1)}{1.2 \cdot \gamma \cdot(\gamma+1)} x^{2}+$ $\frac{\alpha(\alpha+1)(\alpha+2) \beta(\beta+1)(\beta+2)}{1.2 .3 . \gamma(\gamma+1)(\gamma+2)} x^{3}+\cdots . "$ J. Reine Angew. Math., vol. 15, pp. 39-83 and 127-172, 1836.

[10] J. L. Lavoie, F. Grondin, and A. K. Rathie, "Generalizations of Watson's theorem on the sum of a ${ }_{3} F_{2}$." Indian J. Math., vol. 34, no. 1, pp. 23-32, 1992.

[11] J. L. Lavoie, F. Grondin, and A. K. Rathie, "Generalizations of Whipple's theorem on the sum of a ${ }_{3} F_{2}$." J. Comput. Appl. Math., vol. 72, no. 2, pp. 293-300, 1996, doi: 10.1016/03770427(95)00279-0.

[12] J. L. Lavoie, F. Grondin, A. K. Rathie, and K. Arora, "Generalizations of Dixon's theorem on the sum of a ${ }_{3} F_{2}$." Math. Comput., vol. 62, no. 205, pp. 267-276, 1994, doi: 10.2307/2153407.

[13] G. V. Milovanović, R. K. Parmar, and A. K. Rathie, "A study of generalized summation theorems for the series ${ }_{2} F_{1}$ with an applications to Laplace transforms of convolution type integrals involving Kummer's functions ${ }_{1} F_{1}$." Appl. Anal. Discrete Math., vol. 12, no. 1, pp. 257-272, 2018.

[14] H. Prodinger, "Sums of Choi, Zornig, and Rathie - an elementary approach." Quaest. Math., vol. 32, no. 2, pp. 265-267, 2009, doi: 10.2989/QM.2009.32.2.8.801.

[15] A. P. Prudnikov, Y. A. Brychkov, and O. I. Marichev, Integrals and series. Volume 3: More special functions. Transl. from the Russian by G. G. Gould. New York: Gordon and Breach Science Publishers, 1990.

[16] E. D. Rainville, "Special functions." New York: The Macmillan Company. XII, 365 p. (1960)., 1960.

[17] M. A. Rakha and A. K. Rathie, "Generalizations of classical summation theorems for the series ${ }_{2} F_{1}$ and ${ }_{3} F_{2}$ with applications." Integral Transforms Spec. Funct., vol. 22, no. 11, pp. 823-840, 2011, doi: 10.1080/10652469.2010.549487.

[18] H. M. Srivastava, "Sums of a certain family of series." Elem. Math., vol. 43, no. 2, pp. 54-58, 1988.

[19] H. M. Srivastava, "Sums of a certain class of q-series." Proc. Japan Acad., Ser. A, vol. 65, no. 1, pp. 8-11, 1989, doi: 10.3792/pjaa.65.8.

[20] M. Vowe and H.-J. Seiffert, "Aufgabe 946.” Elem. Math., vol. 42, no. 4, pp. 111-112, 1987.

\section{Authors' addresses}

\section{Sungtae Jun}

General Education Institute, Konkuk University, Chungju 380-701, Korea

E-mail address: s jun@kku.ac.kr 
Gradimir V. Milovanović

Serbian Academy of Sciences and Arts, 11000 Belgrade, Serbia, \&, Faculty of Sciences and Mathematics, University of Niš, 18000 Niš, Serbia

E-mail address: gvmemi.sanu.ac.rs

\section{Insuk Kim}

Department of Mathematics Education, Wonkwang University, Iksan 570-749, Korea

E-mail address: iki@wku.ac.kr

\section{Arjun K. Rathie}

Department of Mathematic, Vedant College of Engineering and Technology (Rajasthan Technical University), Bundi, Rajasthan, India

E-mail address: arjunkumarrathie@gmail.com 